\title{
Quantification of Diabetic Macular Ischemia: Assessing Agreement and Reproducibility Between Optical Coherence Tomography Angiography and Fluorescein Angiography
}

\author{
Jeniffer Trenado Luna ${ }^{1}$, Sergio Rojas Juárez ${ }^{1}$ \\ ${ }^{1}$ Hospital de la Luz, Ezequiel Montes 135 Tabacalera Mexico City, 06030, Mexico
}

\begin{abstract}
Introduction: The purpose was to assess the level of agreement and reproducibility between fluorescein angiography (FA) and optical coherence tomography angiography (OCTA), throughout the evaluation of the foveal avascular zone (FAZ) to diagnose diabetic macular ischemia (DMI).

Methods: In this ambispective, observational, cross-sectional research, 52 eyes underwent traditional FA and OCTA to obtain the level of agreement and reproducibility between them, using an intraclass correlation coefficient (ICC) in mixed models; for this purpose, two raters independently graded the area of the FAZ using the OCTA Spectralis and the ImageJ software. Additionally, Spearman and rank-biserial correlational analyses were also realized to calculate the strength of linear relationship between the area of the retinal vessels, the thickness of subfoveal retinal and choroid layers, the presence of neovascularization, the visual acuity, and the grade of diabetic retinopathy using the grades of the Early Treatment Diabetic Retinopathy Study protocols.
\end{abstract}

Result: From the evaluated eyes, the level of agreement between the OCTA and FA among the raters had an ICC of 0.99 and 0.907 ( $p<.001)$, respectively. Furthermore, the reproducibility analysis had an ICC of 0.85 ( $p<.001)$. In the correlation analyses, the enlargement of the FAZ was associated with a decrease in the outer retinal layers $(r=-0.458, p<0.001)$, the photoreceptor layer $(r=-0.32, p=0.021)$, their outer segments $(r=-0.32, p=0.021)$ and the subfoveal choroidal thickness $(r=-0.483, p<0.001)$. $A$ reduction of the vascular area was also observed in higher grades of diabetic retinopathy ( $r=-$ $0.395, p=0.38)$.

Conclusion: Compelling evidence of a high level of agreement and reproducibility between OCTA and FA was obtained to diagnose diabetic macular ischemia. Moreover, it is suggested that the increase in macular ischemia leads to the thinning of the photoreceptor, outer retinal and choroid layers.

\footnotetext{
Keywords: Diabetic macular ischemia, diabetic retinopathy, foveal avascular zone, optical coherence tomography angiography, fluorescein angiography.

Cite This Article: TRENADO, jeniffer; ROJAS JUÁREZ, sergio. Quantification of diabetic macular ischemia:

Assessing agreement and reproducibility between Optical coherence tomography angiography and

Fluorescein angiography. International Journal of Retina, [S.I.], v. 3, n. 1, feb. 2020. ISSN 2614-8536.

Available at: https://www.ijretina.com/index.php/ijretina/article/view/101 Date accessed: 21 feb. 2020. doi:

https://doi.org/10.35479/ijretina.2020.vol003.iss001.101
}

*Correspondence to: Jeniffer Trenado Luna, Hospital de la Luz Ciudad de México, México, jluna91@gmail.com

\section{INTRODUCTION}

Diabetic macular ischemia (DMI) is a significant cause of visual loss in patients with diabetic retinopathy. ${ }^{1}$ The reported prevalence of DMI is approximately $7 \%$ in clinical trials. ${ }^{2}$ The diagnosed is still based on the size and appearance of the foveal avascular zone (FAZ) using a Fluorescein Angiography (FA) 
and it is characterized by its increment, the disrupted margins and the loss or abnormalities of the surrounding retinal capillaries. ${ }^{3,4}$ Previous studies have revealed the relevance of macular ischemia, defined in report 11 of the Early Treatment Diabetic Retinopathy Study (ETDRS), as an essential predictor on the prognosis of patients with diabetic retinopathy $(D R){ }^{5}$ The relationship between $\mathrm{DMI}$ and capillary closures surrounding the FAZ has already been tested, but the vessels in other retinal areas have yet to be measured. ${ }^{2}$ Nonetheless, the caliber of the retinal vessels is a marker for the presence, or severity, of diseases like DR, glaucoma or systemic hypertension. ${ }^{6,7}$ Moreover, Byeon, et al., (2009) ${ }^{8}$ have reported damage to the ganglion cell layer in the fovea and the thinning of the inner retinal layers in patients with DMI.

The characteristics of the FAZ were established since 1980 on the report number 11 of the ETDRS. Since then, numerous studies have shown an association between the presence of $\mathrm{DMI}$ and visual loss. Other studies have suggested the association of DMI with adverse or diminished effects on outcomes of patients with macular edema, regardless whether they were treated with intravitreal therapies or laser photocoagulation. ${ }^{4,9}$

FA has been the gold standard for the diagnosis and staging of diabetic retinopathy since $1961 .^{10}$ However, it requires specific supplies, trained personnel and it is a time-consuming method, requiring up to 10 minutes for acquisition. ${ }^{11}$ Moreover, it is an invasive technique that involves venipuncture of dye with documented side effects like nausea, vomiting, yellow pigmentation of skin and urine, and, in rare cases, anaphylaxis and death. ${ }^{10}$

The recent development of Optical Coherence Tomography Angiography (OCTA) offers an alternative to $F A$, as a noninvasive three-dimensional cross-sectional imaging of retinal vasculature. It allows the detection of the retina and choroidal structures by motion contrast and high-speed scanning, comparing the decorrelation signal between multiple sequential B-scans, and assuming that the only moving aspect is the vascular flow. The resulting decorrelation map is a reconstruction of the blood vessels with erythrocytes flowing into them. ${ }^{12,13}$ OCTA facilitates the accurate diagnosis and follow-up of retinal vascular diseases, as morphological and perfusion characteristics can be obtained instantaneously. Furthermore, early changes provoked by retinal diseases, as remodeling of the FAZ, vascular tortuosity, narrowing of capillary lumen and capillary dilation, cannot be seen in the FA as in the OCTA. ${ }^{11}$

Although the clinical application of OCTA is still limited in DMI by factors like segmentation errors, and the lack of ideal automatic algorithms to resolve these, the need for good patient fixation, sensitivity to minor eye movements, the lack of optimal algorithms that correct these motion artefacts, and discrepancy between measurements using different commercial devices, suggests that OCTA may be the gold standard for DMI within the next few years. ${ }^{14}$

Therefore, the purpose of this study was to assess the level of agreement and reproducibility between FA and OCTA to evaluate the FAZ in patients with diabetic macular ischemia. It is worth mentioning that studies like Bradley P.D. et al., (2016) ${ }^{15}$, used a weighted kappa ( $\mathrm{K}$ ) analysis for the level of agreement ${ }^{16}$, basing the results upon the ordinal property of its variables: the ETDRS's scores. In this study we analyzed the FAZ area as a continuous variable using the intraclass correlation coefficient, so the level of agreement was independent from the ETDRS scores, therefore, resulting in a more reliable and objective analysis.

Moreover, unlike Botto de Barros-G J.M. et al., $(2016)^{10}$ or La Mantia A., et al., (2019) ${ }^{12}$, 
that only compared the FA with the OCTA, this study included vertical measurements of the different retinal layers and their relationship with macular ischemia, allowing a multi-dimensional analysis of agreement between techniques and topographical correlations with DMI within the same sample.

\section{METHODS}

This was an observational, ambispective, crosssectional study approved by the institutional review board, and was executed in compliance with the tenets of the Declaration of Helsinki. Signed informed consent was obtained from each patient prior to enrollment. The sample consisted of patients' eyes with any stage of diabetic retinopathy, though they were categorized based on their retinopathy status. The images were collected as patients underwent traditional FA and OCTA over an eight months period; from May 1, to December 20, 2018. All medical records from patients were retrospectively reviewed and completed in the following visits. Exclusion criteria comprehended the presence of macular edema caused by other diseases, such as epiretinal membrane, congenital maculopathies, age-related macular degeneration, scarring of any etiology, venous or arterial occlusions, or subjects who presented motion artifacts or poor signal strength; patients with significant cataracts were also excluded.

\section{Acquisition of fundus fluorescein angiograms}

All FA images were acquired with a digital retinal camera system (Topcon TRC-50DX; Topcon Medical Systems Inc., Paramus, NJ, USA), centered on the macula, 20-40 seconds after the contrast injection in the arteriovenous phase, and $40+$ seconds in the later phases. All images were performed by a single experienced technician (Olivares, T) between 9:00 AM and 3:00 PM.

\section{Acquisition of OCTA images}

After the acquisition of the FA images, the patients with DMI diagnosis, corroborated by a retina specialist, were contacted on the same day of the FA pictures, and were requested to assist within a maximum period of one week to acquire the OCTA images, by a single experienced technician (Olivares, T). The superficial vascular plexus on the OCTA (Spectralis ${ }^{\circledR}$, version 1.10.2.0, HRA-OCT; Heidelberg Engineering; Germany) was analyzed using an image size of $3 \times 3 \mathrm{~mm}$ composed of $512 \times 512$ scans, centered on the fovea. The scale is embedded within the OCTA Spectralis software.

\section{Quantification of the FAZ}

Using the acquired images, two graders (T.J. and R.S.) performed measurements of the FAZ. OCTA images at the levels of superficial capillary networks and FA images at the arteriovenous phases were loaded into ImageJ software, an image analysis program (Rasband, W.S., ImageJ 1.52a, U. S. National Institutes of Health, Bethesda, Maryland, USA, https://imagej.nih.gov/ij/, 1997-2018). Image brightness and contrast were modified to delimit the FAZ. The scale for the FA images was based on the diameter of the optic nerve of each image, with an approximate diameter of $1.5 \mathrm{~mm}$. The scale for the OCTA images in the ImageJ software considered the 200 microns scale bar embedded in the lower-left corner of each $3 \times 3 \mathrm{~mm}$ OCTA Spectralis image. ${ }^{17-19}$ The area of the FAZ and maximum horizontal and vertical diameters were then marked manually as shown in [Figure 1]. The graders performed the assessments independently, using protocols from ETDRS report No. 11 and $19^{4,20}$ and were masked to the results of each other. In case of disagreement between them, open adjudication was used to resolve the final grading decision. Questionable DMI grades were excluded. 


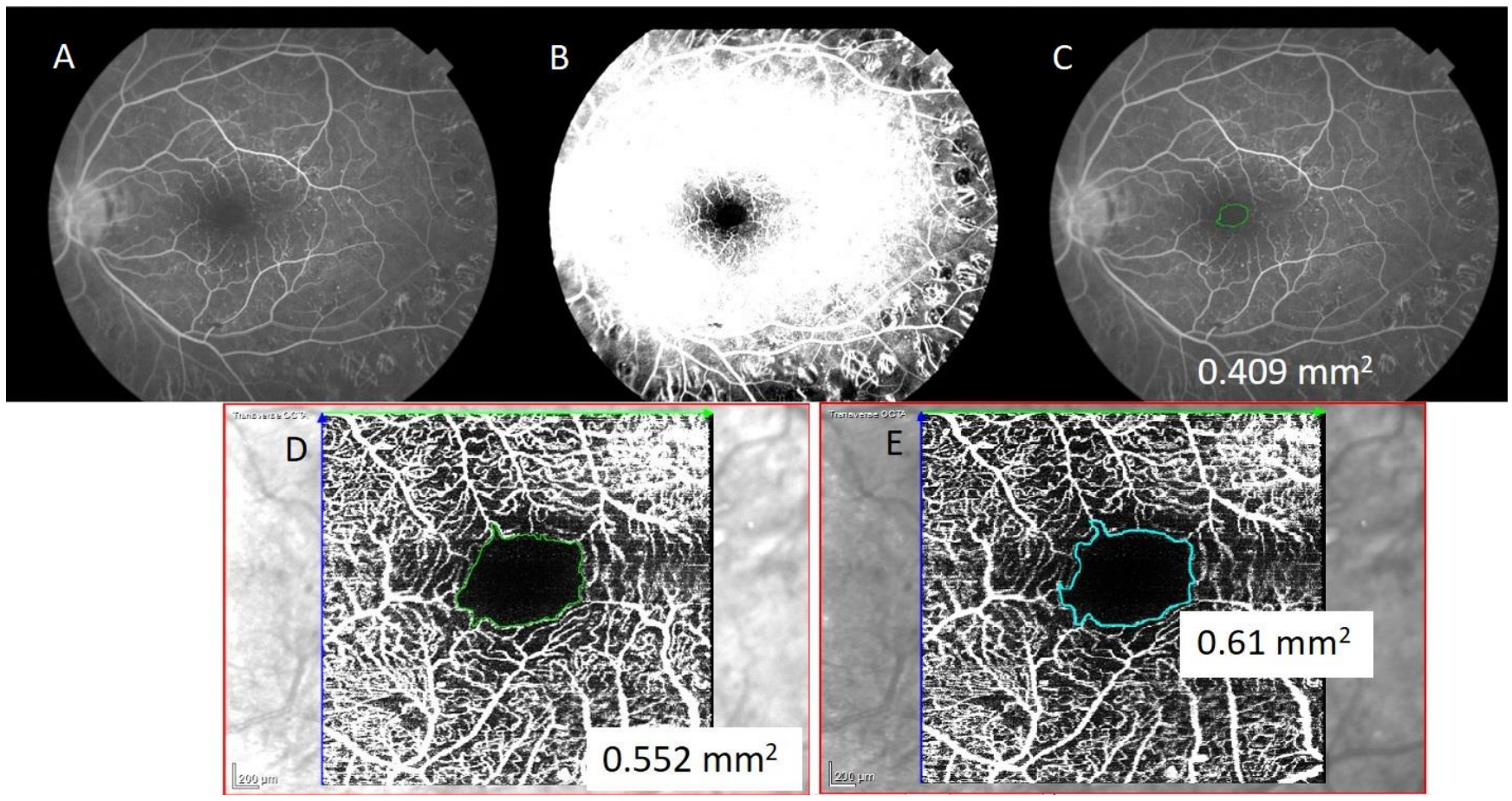

Figure 1. Diabetic macular ischemia as seen on FA versus OCTA. Evaluation of FAZ in FA compared to OCTA. FAZ areas on both methods are represented by green colored line. (A) Demonstrating the presence of macular ischemia in FA. (B) Demonstrating the change of contrast and brightness of colors in ImageJ to facilitate the measurement of the FAZ. (C) Green colored line delimits FAZ in FA. (D) Green colored line delimits FAZ in OCTA measured in ImageJ. (E) Blue colored line delimits FAZ measured in OCTA Spectralis.

\section{Choroidal and retinal layers thickness}

A Spectral Domain Optical Coherence Tomography Angiography (Spectralis; Heidelberg Engineering, Germany) was used to perform a $30 \times 15^{\circ}(\sim 8.8 \times 4.4 \mathrm{~mm})$ scan pattern centered on the fovea. The perpendicular distance from the bottom of the RPE-Bruch's membrane complex to the chorioscleral junction was recorded as the subfoveal choroidal thickness, using the caliper tool available with the proprietary OCT viewing software. The central subfield thickness and the subfoveal volume were assessed using automatic measured values. The retinal layers were evaluated with the OCTA images acquired previously, and each layer was measured with the ImageJ software caliper tool. [Figure 2]

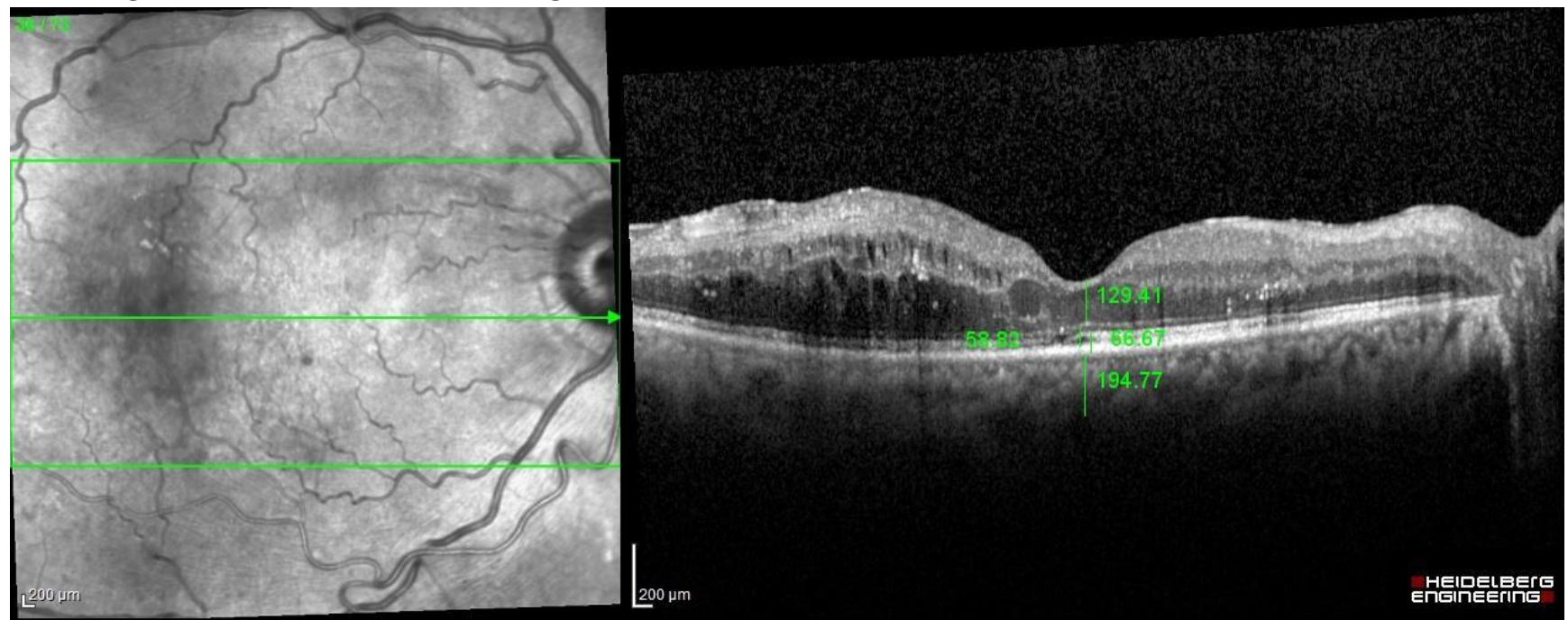

Figure 2. Macular B-scan with the measurements of the retinal layers. 


\section{Area of the retinal vessels}

The area of the FAZ in each image obtained with the OCTA, in a $3 \times 3 \mathrm{~mm}$ square next to the optic disc, was obtained and measured after the skeletonization of the vessels with the ImageJ software.

\section{Statistical Analysis}

All the data analysis was processed in SPSS (IBM Corp. Released 2015. IBM SPSS Statistics for Windows, Version 23.0. Armonk, NY: IBM Corp.). For the sample, a non-probability convenience sampling technique was used. Also, the data was evaluated with D'Agostino K2 test to discard normal distribution before applying the respective statistical tests, and a $\mathrm{P}$ value of $<0.05$ was considered statistically significant. Moreover, the values that were two standard deviations away from the mean were considered outliers; that means that $2.3 \%$ of the values in the higher and lower ends of the spectrum of the normal distribution were discarded by this cutoff. To assess the level of agreement and reproducibility, a two-way, intraclass correlation coefficient (ICC) of absolute agreement and single rating, in mixed models, was calculated. The Spearman and rank-biserial correlational analyses were used to evaluate the strength of the linear relationship between the variables.

\section{RESULTS}

The study included 75 eyes with DMI. Nonetheless, after the cutoff, the sample consisted of only 52 eyes. According to the number of eyes, we obtained a margin of error of $11.32 \%$ with a $95 \%$ confidence interval. Of these patients, $41.46 \%(\mathrm{~N}=17)$ were female and $58.54 \%(\mathrm{~N}=24)$ male. The mean patient age was $64.57 \pm 8.07$ years, and the mean time for diabetic evolution was $17.23 \pm 6.09$ years. All eyes had diabetic retinopathy; 31 (59.61\%) had DR modified by laser without neovascular activity, 6 (11.53\%) proliferative diabetic retinopathy (PDR) without high risk characteristics, 5 (9.61\%) very severe non proliferative diabetic retinopathy (NPDR), 5 (9.61\%) DR modified by laser with neovascular activity, and 5 (9.61\%) had severe NPDR [Tab. I].

Table I. Demographic and clinical characteristics of patients with diabetic macular ischemia in OCTA data

\begin{tabular}{|c|c|c|c|c|c|}
\hline \multirow{2}{*}{ Parameters } & Severe NPDR & $\begin{array}{c}\text { Very severe } \\
\text { NPDR }\end{array}$ & $\begin{array}{c}\text { PDR without } \\
\text { HR }\end{array}$ & $\begin{array}{c}\text { DR modified by } \\
\text { laser w/o NA }\end{array}$ & $\begin{array}{l}\text { DR modified by } \\
\text { laser w/NA }\end{array}$ \\
\hline & (5 eyes) & (5 eyes) & (6 eyes) & (31 eyes) & (5 eyes) \\
\hline \multicolumn{6}{|l|}{ Age } \\
\hline Mean & 64.2 & 70.6 & 62.7 & 62.9 & 57.6 \\
\hline Median & 65 & 73 & 64 & 62 & 57 \\
\hline Standard Dev. & 10.28 & 3.91 & 2.07 & 9.07 & 3.78 \\
\hline Sex Ratio (M: F) & $4: 1$ & $3: 2$ & $0: 6$ & $18: 31$ & $2: 3$ \\
\hline
\end{tabular}

NPDR=non proliferative diabetic retinopathy; $\mathrm{PDR}=$ proliferative diabetic retinopathy; $\mathrm{HR}=$ high risk, NA=neovascular activity; DR=diabetic retinopathy. 
The level of agreement in the OCTA and FA between raters had an ICC of 0.99 and 0.907 respectively $(p<.001)$. In the reproducibility analysis between the studies, an ICC of $0.85(p<.001)$ was found.

For the statistical analysis, the data of the rater with the highest consistency $(0.897, p<.001)$ was used according to the ICC. According to the OCTA and FA, the mean FAZ was $0.629 \pm 0.459$ and $0.613 \pm 0.412$ $\mathrm{mm}^{2}$, respectively.

Based on the OCTA, one eye (2\%) was identified with no macular ischemia, five eyes (10\%) as grade one of macular ischemia, 36 eyes (69\%) as grade two, and 10 eyes (19\%) as grade three. Similar results were obtained using the FA; six eyes (12\%) presented grade one, 37 eyes (71\%) grade two, and nine (17\%) grade three.

Linear correlation plots between the enlargement of the $F A Z$, the outer retinal layers height $(r=-0.458$, $\mathrm{p}=0.001)$, the photorreceptor layers $(r=-0.32$, $p=0.021)$, their outer segments $(r=-0.32, p=0.021)$, and the choroidal thickness $(r=-0.483, p<.001)$ showed a progressive decline (Figs. 3-5). The presence of greater grade of DR was associated with the decrease of the outer retinal layers $(r=-0.5$, $\mathrm{p}=0.048)$. The inner retinal layers were not significantly correlated. [Figures 3-5]

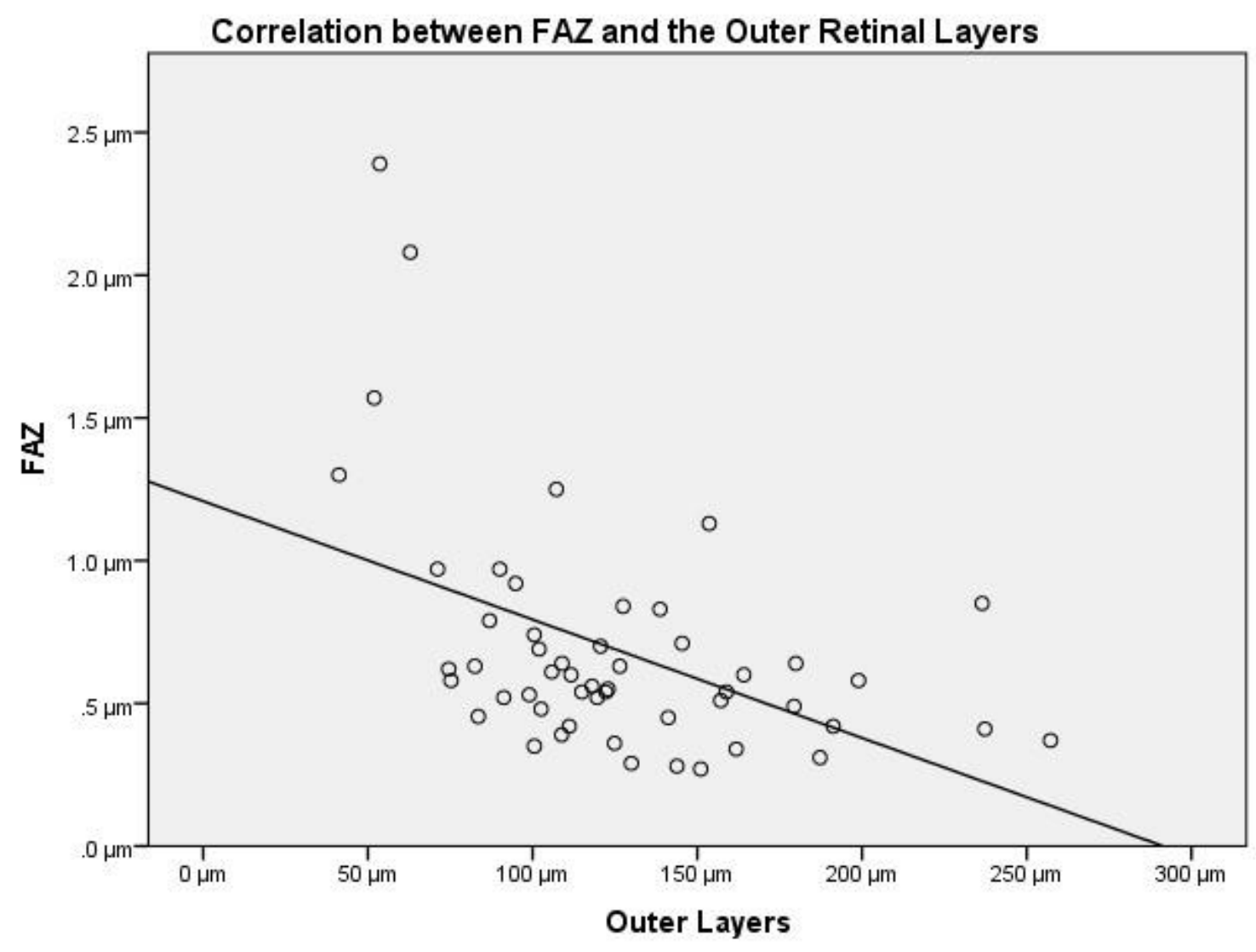

Significant correlation at .01 (bilateral); $1=-.458, p=.0006$

Figure 3. Correlation between FAZ and the Outer Retinal Layers. Scatterplot of the FAZ area and the outer retinal layers showing the negative relationship between the variables in eyes with DMI. 


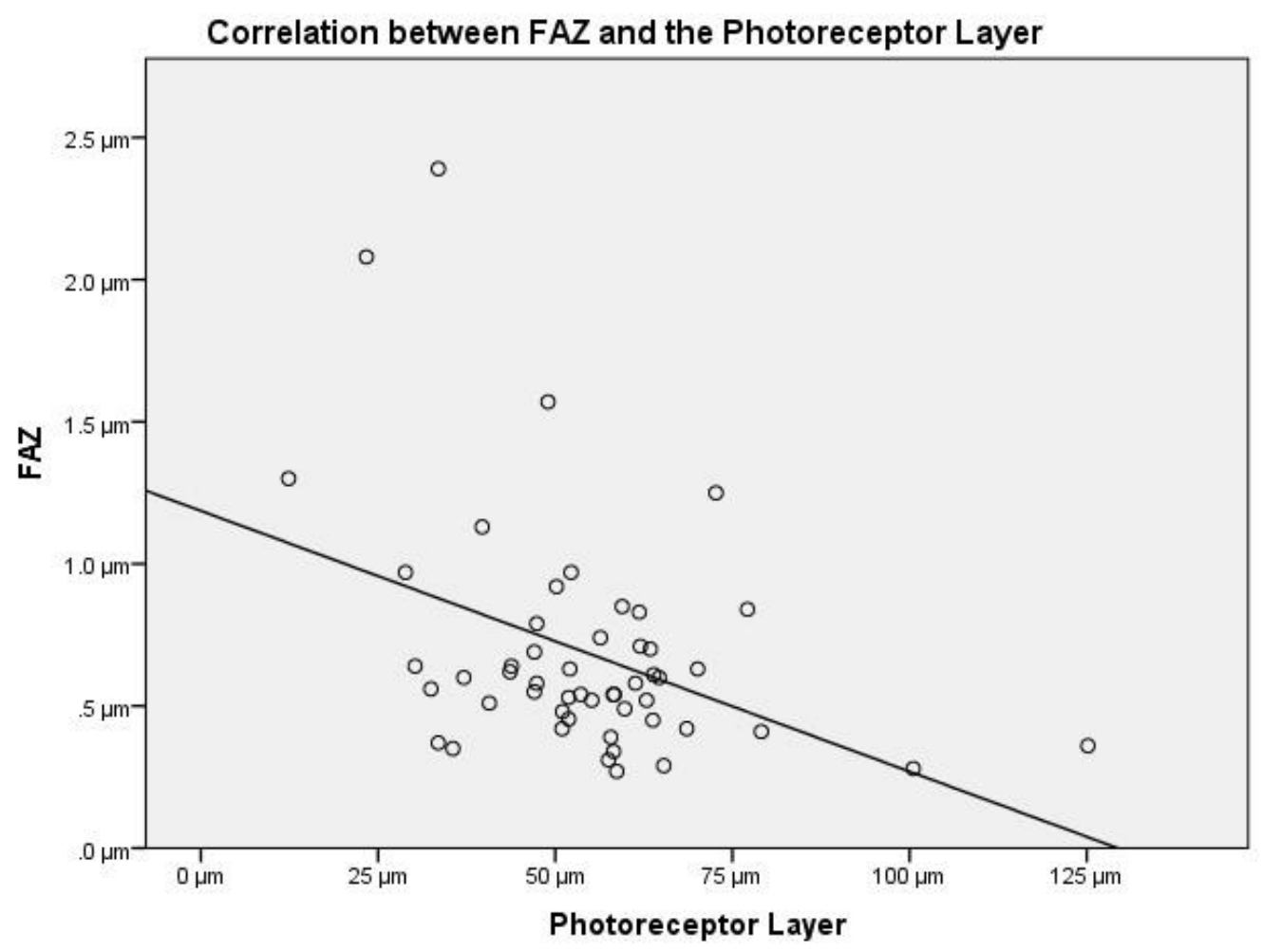

Significant correlation at .05 (bilateral); $1=-.32, p=.021$

Figure 4. Correlation between FAZ and the Photoreceptor Layer. Scatterplot of the FAZ area and the photoreceptor layer showing the negative relationship between the variables in eyes with DMI.

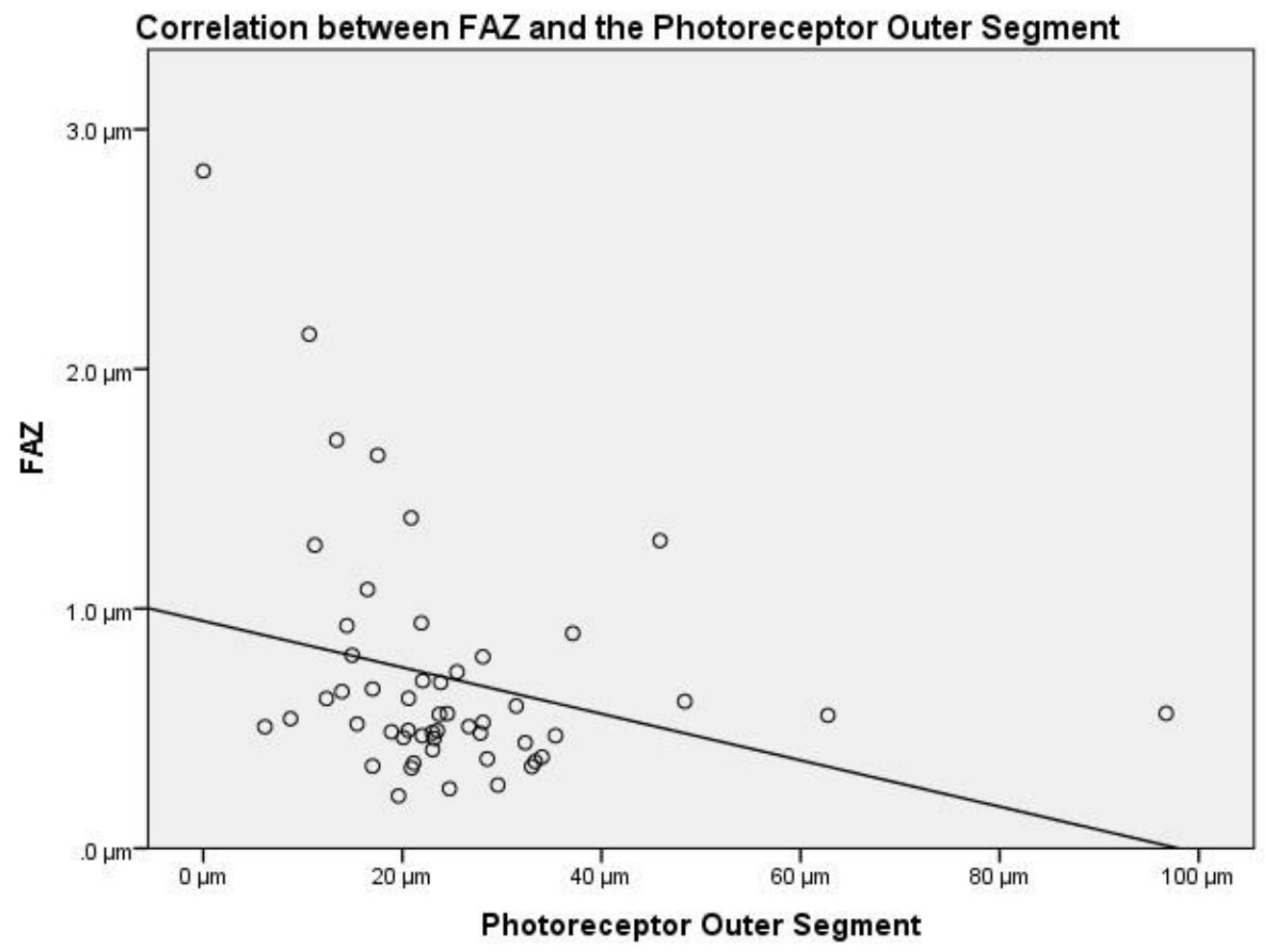

Significant correlation at .05 (bilateral); $1=-.32, p=.021$

Figure 5. Correlation between FAZ and the Photoreceptor Outer Segment. Scatterplot of the FAZ area and the photoreceptor outer segment layer showing the negative relationship between the variables in eyes with DMI. 
The results provided in this study yield useful information about the area of the retinal vessels obtained by ImageJ, observing a $2.681 \pm 0.815 \mu \mathrm{m}^{2}$. A smaller vessel area was observed in relation to a greater grade of DR $(r=-0.395, p=0.38)$ and a thicker choroid layer $(r=-0.461, p=0.014)$. Finally, the outer retinal layers seemed to decrease as the retinal neovascularization increases $(r=-0.331, p=0.016)$.

\section{DISCUSSION}

The recent use of the OCTA allows the visualization of the retinal layers, its capillaries and the construction of microvascular flow maps. According to the high level of agreement between the OCTA and the FA, and the high degree of reproducibility and interrater agreement acquired by OCTA, we suggest that the OCTA may be used for the diagnosis of diabetic macular ischemia, once validated in a larger population. ${ }^{11,21}$

The grade of macular ischemia contributes to DR worsening; even more than systemic factors, especially over a short time of period. ${ }^{22}$ Several studies have shown changes in the retinal layers; Byeon et al., (2009) $)^{8}$ and Liew G. et al., (2015) ${ }^{6}$ have reported damage to the foveal ganglion cell layer and loss of the inner retinal layers, by a SD-OCT, corresponding to the area of reduction of capillary perfusion. Other studies have reported thickening of the ischemic retina, especially in the intermediate layers. ${ }^{2}$ Sim D.A., et al., $(2014)^{23}$ found thinning of the retinal nerve fiber layer, outer retina, and thickening of Haller's large vessel layer of the choroid.

In this study, a grater grade of DMI was found in association with a decrease in the outer retinal layers, the photoreceptor layer, their outer segments, and the choroidal thickness. The increase in the FAZ induces a disruption of the outer limiting membrane and the photoreceptor complex, as well as a thinning of the outer layers. The inner retinal layers were not statistically significantly affected. These differences could be related to the diabetic evolution time, given that chronic isquemia may cause retinal atrophy and a reduction of the foveal volume in advanced stages. 3 Other mechanisms, such as the damage to the hematoretinal barrier, increased extravasation of inflamatory cells, hard foveal exudates that lead to fibrosis, and cystic spaces in macular edema in the outer and inner photoreceptor segments, also contribute to the damage. ${ }^{24}$

Kifley A. et al., (2007) ${ }^{7}$ and Klein R. et al., (2012) ${ }^{25}$ showed the association between wider diameter venules and the progression of diabetic retinopathy, implying that the endothelial dysfunction, inflammatory changes, and hyperglycemia may be the cause. Though this study considered both venules and arterioles, our results strongly suggest microvascular affection in $\mathrm{DR}$, as a reduction of the vascular area observed in patients with higher grade of DR. Similar results were obtained by Scarinci F. et al., (2015) $)^{3}$ who associated arteriolar narrowing with higher grades of ischemia.

Our study had its limitations; further studies should be performed with a bigger sample to provide additional support to these results. OCTA requires patient fixation for several seconds; due to this fact, our interpretation of some images was limited by a segmentation artifact. There is also a confounding factor that contributes to the ischemic status, such as previous laser treatment, anti-VEGF injections, and the duration of macular edema. Some images could also be limited by the presence of edema that masked the flow signal from the perfused vessels and may modified the data interpretation. Despite the use of a software to perform the measurements, there is still an operator dependent factor that should be taken into account. Although the clinical application of OCTA is limited in DMI by the factors mentioned above, overall, traditional FA is more sensitive in identifying early signs of diabetic retinopathy and lesions with low flow characteristics. 
Moreover, the FA can capture a much wider area of the retinal vasculature, its images are less liable to show artifacts than the OCTA and are easier to interpret. Nonetheless, this study allowed us to establish the probability of achieving an accurate diagnosis of DMI using OCTA.

Furthermore, the OCTA with the support of the software ImageJ, allow to propose a new set of criteria for the quantitative measurement of the $F A Z$, based on the ETDRS, as grade $0:<0.196 \mathrm{~mm}^{2}$ (250 $\mu \mathrm{m}$ radius), grade $1: \geq 0.196 \mathrm{~mm}^{2}$ (250 $\mu \mathrm{m}$ radius) $<0.282 \mathrm{~mm}^{2}$ (300 $\mu \mathrm{m}$ radius), grade $2: \geq 0.282 \mathrm{~mm}^{2}$ (300 $\mu \mathrm{m}$ radius) $<0.785 \mathrm{~mm}^{2}$ (500 $\mu \mathrm{m}$ radius), grade 3: $\geq 0.785 \mathrm{~mm}^{2}$ (500 $\mu \mathrm{m}$ radius) and grade 8 : cannot grade. However, this finding should be developed in future research.

\section{CONCLUSIONS}

A high level of agreement was obtained between diabetic macular ischemia grading results for OCTA and conventional FA, using standard ETDRS protocols; so it may be used for the diagnosis of diabetic macular ischemia. Evidence that the increase in macular ischemia leads to thinning of the photoreceptors and the outer retinal layers was also found.

The OCTA has the potential to change the way we detect retinal diseases; it could reduce the number of indications of conventional FA and potentially improve clinical efficiency, by reducing the time consumed to perform an FA providing consistent and reproducible results. However, further research is required before OCTA is established as the main tool used to diagnose DMI through the FAZ.

\section{ACKNOWLEDGEMENTS}

The authors thank Alejandro Macías for the software and technical support, Héctor Pérez Cano for the collaboration in the review of the article, and the Hospital de la Luz for facilitating the equipment for the images.

\section{REFERENCES}

1. Lee D.H., Kim J.T., Jung D.W., Joe S.G., Yoon Y.H. Relationship between foveal ischemia and spectral-domain optical coherence tomography findings in ischemic diabetic macular edema. IOVS. 2013; 54(2): p. 1080-1085.

2. Liew G., Sim D.A., Keane P.A., Tan A.G., Mitchell P., Wang J.J., Wong T.Y., Fruttiger M., Tufail A., Egan C.A. Diabetic macular ischemia is associated with narrower retinal arterioles in patients with type 2 diabetes. Acta Ophthalmol. 2015; 93: p. e45-e51.

3. Pereira, F., Godoy, B.R., Maia, M., Regatieri, C.V. Microperimetry and OCT angiography evaluation of patients with ischemic diabetic macular edema treated with monthly intravitreal bevacizumab: a pilot study. Int J Retin Vitr. 2019; 5: 24 .

4. Scarinci F., Jampol L.M., Linsenmeier R.A., Fawzi A.A. Association of diabetic macular nonperfusion with outer retinal disruption on optical coherence tomography. JAMA Ophthalmol. 2015; 133(9): p. 1036-1044.

5. Early Treatment Diabetic Retinopathy Study Research Group. Classification of diabetic retinopathy from fluorescein angiograms: ETDRS report number 11. Ophthalmology. 1991; 98: p. 807-822.

6. Usman, M. An overview of our current understanding of diabetic macular ischemia. Cureus. 2018; 10(7): e3064.

7. Kifley A., Wang J.J., Cugati S., Wong T.Y., Mitchell P. Retinal vascular caliber, diabetes, and retinopathy. Am J Ophthalmol. 2007; 143(6): p. 1024-1026.

8. Byeon S.H., Chu Y.K., Lee H., Lee S.Y., Kwon O.W. Foveal ganglion cell layer damage in ischemic diabetic maculopathy: Correlation of optical coherence tomography and anatomic changes. Ophthalmology. 2009; 116: p. 1949-1959. 
9. Sim D.A., Keane P.A., Zarranz-Ventura J., Fung S., Powner M.B., Platteau E., Bunce C.V., Fruttiger M., Patel P.J., Tufail A., Egan C.A. The effects of macular ischemia on visual acuity in diabetic retinopathy. IOVS. 2013; 54(3): p. 2353-2360.

10. Botto de Barros-G J.M., Toledo-Lima T., Noguera-Louzada R., Thome-Rassi A., CruvinelIssac D.L., Avila M. Diabetic macular ischemia diagnosis: Comparison between Optical Coherence Tomography Angiography and Fluorescein Angiography. J Ophthalmol. 2016; 3989310.

11. Enders C., Baeuerlea F., Lang G.E., Dreyhauptb J., Lang G.K., Loidl M., Werner J.U. Comparison between findings in Optical Coherence Tomography Angiography and in Fluorescein Angiography in patients with diabetic retinopathy. Opthalmologica. 2019; 28: p. 1-6.

12. La Mantia A., Kurt R.A., Mejor S., Egan C.A., Tufail A., Keane P.A., Sim D.A. Comparing fundus Fluorescein Angiography and Swept-source Optical Coherence Tomography Angiography in the evaluation of diabetic macular perfusion. Retina. 2019; 39(5): p. 926-937.

13. Sourour O., Arya M., Waheed N. New findings and challenges in OCT angiography for diabetic retinopathy. Ann Eye Sci. 2018; 3: 44.

14. Khadamy J.; Aghdam K.A., Falavariani K.G. An update on Optical Coherence Tomography Angiography in diabetic retinopathy. J Ophthalmic Vis Res. 2018; 13(4): p. 487-497.

15. Bradley P.D., Sim D.A., Keane P.A., Cardoso J., Agrawal R., Tufail A., Egan C.A. The evaluation of diabetic macular ischemia using optical coherence tomography angiography. IOVS. 2016; 57(2): p. 626-631.

16. Ranganathan P., Pramesh C.S., Aggarwal R. Common pitfalls in statistical analysis: Measures of agreement. Perspect Clin Res. 2017; 8(4): p. 187-191.
17. Garcia-Garrido M., Mühlfriedel R.L., Beck S.C., Wallrapp C., Seelige M.W. Scale Adjustments to Facilitate TwoDimensional Measurements in OCT Images. PloS one. 2015; 10(6): e0131154.

18. Shahlaee A., Pefkjanaki M., Hsu J., Ho A.C. Measurement of foveal avascular zone dimensions and its reliability in healthy eyes using Optical Coherence Tomography Angiography. Am J Ophthalmol. 2016; 161: p. 50-55. e1.

19. Araki S., Miki A., Goto K., Yamashita T., Yoneda T., Haruishi K., leki Y., Kiryu J., Maehara G., Yaoeda K. Foveal avascular zone and macular vessel density after correction for magnification error in unilateral amblyopia using optical coherence tomography angiography. BMC ophthalmol. 2019; 19(1): 171.

20. Early Treatment Diabetic Retinopathy Study Research Group. Focal photocoagulation treatment of diabetic macular edema. Relationship of treatment effect to fluorescein angiographic and other retinal characteristics at baseline: ETDRS report no. 19. Arch Ophthalmol. 1995; 113: p1144-1155.

21. Samara W., Shahlaee A., Adam M.K., Khan M.A., ChiangA., Maguire J.I., Hsu J., MD, Ho A.C. Quantification of Diabetic Macular Ischemia Using Optical Coherence Tomography Angiography and Its Relationship with Visual Acuity. Ophthalmology. 2017; 124(2): p. 235-244.

22. Ip M., Domalpally A., Sun J., Ehrlich J. Long-term effects of therapy with ranibizumab on diabetic retinopathy severity and baseline risk factors for worsening retinopathy. Ophthalmology. 2015; 122(2): p. 367-374.

23. Sim D.A., Keane P.A., Fung S., Karampelas M., Sadda S.R., Fruttiger., Patel P.J., Tufail A., Egan C.A. Quantitative Analysis of Diabetic Macular Ischemia Using Optical Coherence Tomography. IOVS. 2014; 55: p. 417-423. 
24. Murakami T., Nishijima K., Akagi T., Uji A., Horii T., Ueda-Arakawa N., Muraoka Y.,Yoshimura N. Optical Coherence Tomographic Reflectivity of Photoreceptors beneath Cystoid Spaces in Diabetic Macular Edema. IOVS. 2012; 53: p. 1506-1511.

25. Klein R., Myers C.E., Lee K.E., Gangnon R., Klein B.E. Changes in retinal vessel diameter and incidence and progression of diabetic retinopathy. Arch Ophthalmol. 2012; 130(6): p. 749-755.

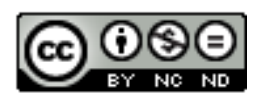

This work licensed under Creative Commons Attribution 